\title{
ERGONOMÍA DEL PUESTO DE TRABAJO DEL PRINCIPIO DE PREVENCIÓN DE LA LEY N 29783 Y SATISFACCIÓN LABORAL DEL PERSONAL ADMINISTRATIVO DE LA SEDE RECTORADO DE LA UNIVERSIDAD PRIVADA DE TACNA, 2018
}

ERGONOMICS OF THE WORKPLACE OF THE PREVENTION PRINCIPLE OF LAW No. 29783 AND THE JOB SATISFACTION OF THE ADMINISTRATIVE PERSONNEL OF THE HEADQUARTERS OF THE UNIVERSITY PRIVATE OF TACNA, 2018

\author{
Elvira Ruth Cabrera Ucharico ${ }^{1}$ \\ Rina María Alvarez Becerra²
}

\begin{abstract}
RESUMEN
El presente estudio indagó la relación entre la ergonomía del puesto de trabajo del Principio de Prevención de la Ley $N^{\circ} 29783$ y su relación con la satisfacción laboral del personal administrativo de la Universidad Privada de Tacna en el 2018. La investigación tuvo una perspectiva cuantitativa, analítica y prospectiva. El diseño fue no experimental, de tipo transeccional, correlacional. Muestra de 61 personal administrativo. Se utilizó el cuestionario de autopercepción de características ergonómicas INSHT y la Escala general de satisfacción laboral. Resultados: 3 de las características ergonómicas del puesto de trabajo como altura del plato de asiento ajustables de 42 a $51 \mathrm{~cm}(\mathrm{p} \mathrm{0,040)}$, borde superior del respaldo al asiento ajustable entre 45 a $55 \mathrm{~cm}$ por encima del asiento $(p 0,04)$ y recomendaciones y goce de descansos frecuentes de minutos cada hora o cada dos horas para los trabajadores que realizan tareas repetitivas durante varias horas $(\mathrm{p} 0,001)$ lo cual dentro del Principio de Prevención de la Ley $\mathrm{N}^{\circ} 29783$ en ciertos casos requiere de corrección, a fin de garantizar los medios y condiciones para proteger la salud y el bienestar de los trabajadores, las mismas que se asociaron estadísticamente con la satisfacción laboral que presenta el personal administrativo de la sede rectorado de la Universidad Privada en Tacna en el 2018.
\end{abstract}

Palabras clave: Ergonomía, Principio de Prevención, Ley $N^{\circ}$ 29783, satisfacción laboral.

\footnotetext{
${ }^{1}$ Maestra en Derecho del Trabajo, Contador Público Colegiado, Auditor Independiente, Docente Universitaria, Asistente en Gestión del Potencial Humano de la Universidad Privada de Tacna. elvira_ruth@hotmail.com, personal@upt.edu.pe. ORCID: 0000-0001-5948-399X

2 Doctora en Educación con mención en Gestión Educativa, Magíster en Docencia Universitaria, Especialista en Estadística de la Investigación, Docente Universitaria, rinaalvarezb@hotmail.com. ORCID: 0000-0002-5455-6632
} 


\begin{abstract}
The present study investigated the relationship between the ergonomics of the workplace of the Prevention Principle of Law No. 29783 and its relationship with the job satisfaction of the administrative staff of the Private University of Tacna in 2018. The research had a quantitative perspective, analytical and prospective. The design was non-experimental, of a transectional, correlational type. Sample of 61 administrative staff. The INSHT ergonomic characteristics self-perception questionnaire and the General Job Satisfaction Scale were used. Results: 3 of the ergonomic characteristics of the workplace such as adjustable seat plate height from 42 to $51 \mathrm{~cm}$ ( $p$ 0.040), upper edge of the backrest to the seat adjustable between 45 to $55 \mathrm{~cm}$ above the seat ( $p$ 0.04) and recommendations and enjoy frequent breaks of minutes every hour or every two hours for workers who perform repetitive tasks for several hours ( $p$ 0.001), which within the Prevention Principle of Law No. 29783 in certain cases requires correction, to In order to guarantee the means and conditions to protect the health and well-being of workers, the same ones that were statistically associated with the job satisfaction presented by the administrative staff of the rector's headquarters of the Private University in Tacna in 2018.
\end{abstract}

Keywords: Ergonomics, Principle of Prevention, Law $N^{\circ} 29783$, job satisfaction

\title{
INTRODUCCIÓN
}

Un antecedente histórico que marca un hito en la concreción de la protección legal de los trabajadores, fue la Asociación Internacional para la Protección Legal de los Trabajadores, fundada en Basilea en 1901, la que recoge los presupuestos del galés Robert Owen y del francés Daniel Legrand, y que más adelante se incorpora en la Constitución de la Organización Internacional del Trabajo (OIT) en 1919, al finalizar la Primera Guerra Mundial en la Conferencia de la Paz en París (Rodríguez,2009).

El Preámbulo de la Constitución de la OIT, instaura los aspectos de la actividad laboral que deben ser mejoradas, los que hasta el día de hoy siguen siendo motivo de reflexión y lucha por parte de la OIT. En esta perspectiva, el Perú como Estado Parte de la Organización Internacional del Trabajo, ha promulgado la Ley $N^{\circ} 29783$ (Contiene un Título Preliminar, 103 artículos, tres disposiciones complementarias finales y siete disposiciones finales ), que plasma la obligación del Estado referida a la protección de los trabajadores. En esta línea discursiva, el contenido del derecho a la vida no se agota en respetar el derecho sino en integrarlo a los derechos económicos y sociales (Mesia,2004). Los hallazgos permiten afirmar que en su mayoría las condiciones ergonómicas del puesto de trabajo cumplen con las características ergonómicas, sin embargo, en muchos casos, se presentan condiciones disergonómicas, que pueden afectar a futuro la salud del personal o exacerbar dolencias existentes, lo cual, requiere de implementar medidas correctivas para materializar el Principio de Prevención que fundamenta la Ley $\mathrm{N}^{\circ} 29783$ y el derecho a la seguridad e Higiene en el Trabajo, establecido en el Pacto Internacional sobre los Derechos Económicos, Sociales y Culturales de 1968, ratificado por el Perú. Fundación Científico Técnica L'Institut 
Sindical de Treball, Ambient i Salut (2011), afirma que, si bien es cierto, la enfermedad y la salud es connatural a la naturaleza humana, no es menos cierto que en el trabajo nos ponemos en relación con exigencias físicas forzadas, materiales, sustancias o con condiciones ambientales y climáticas perjudiciales, etc.". En el Perú , Brunette (2003) afirma que muchas de las condiciones de trabajo son disergonómicas. Se desconoce la magnitud total de población trabajadora que está expuesta a diferentes riesgos ocupacionales.

Mención aparte, merece el constructo satisfacción laboral como reacciones, sentimientos de un miembro de una institución, la cual tendría como presupuesto la ergonomía (Díaz,2014). En este sentido, en el Perú, el Ministerio de Trabajo y Promoción del Empleo del Perú (2009), indicó que a nivel del sector privado, un 6,4\% de los trabajadores en Lima presentan insatisfacción laboral, en la que se evidencia una correlación entre mejores condiciones laborales (condiciones de entorno, servicio y de riesgo) y la mayor satisfacción laboral), sin embargo, se halló que tiene mayor incidencia las condiciones de trabajo (horas de trabajo, ingreso, entre otras) que las condiciones laborales del entorno, de riesgo y de servicio, que para muchos resulta inadecuada y riesgosa.

En el ámbito de estudio, que está referido a la sede rectorado de la Universidad Privada en Tacna, por un lado, se aprecia que con frecuencia el personal evidencia cansancio al inicio de la jornada, expresiones o manifestaciones de expectativas no satisfechas de promociones, o reconocimientos por su trabajo e incluso por la escasa motivación pro parte de sus compañeros o superiores, aunado en muchos casos a trastornos de salud especialmente musculares, cefaleas, cansancio, malestares posturales,afecciones músculo-esqueléticas, condiciones disergonómicas.

En este punto, es posible considerar que quizá la satisfacción laboral esté vinculada con las características ergonómicas del puesto de trabajo. Por tanto, las condiciones de ergonomía no adecuadas podrían suponer un riesgo laboral por labores repetitivas, posturas corporales y disposición del lugar de trabajo inadecuadas y que podría afectar la satisfacción laboral. De otro lado, soslayar las condiciones laborales disergonómicas y la posibilidad de que sean causa de enfermedades profesionales o laborales, implicaría la vulneración del principio de prevención de la Ley $\mathrm{N}^{\circ} 29783$ y su modificatoria por Ley $\mathrm{N}^{\circ} 30222$. Asimismo, el nivel de satisfacción propendería a un mejor o menor rendimiento laboral, razón por la cual, resulta imperativo indagar en aquellos factores que estarían influyendo en la percepción de la satisfacción en el trabajo del personal. Existen casis siempre, problemas de salud relacionados con la carga de trabajo, Andrango y Tituaña (2016). Calderón (2014) en Guayaquil, afirma que la permanencia del personal es un factor de riesgo que podría originar desórdenes traumáticos acumulativos. Rodríguez (2013) en Ecuador manifiesta que el riego ergonómico se encuentra presentes en todas las oficinas donde laboran el personal designado para cumplir tareas de secretaría, por este motivo existen frecuentes absentismos al sufrir dolencias en su salud. León (2013) no encontró una correlación entre factores ergonómicos y el factor extrínseco de la satisfacción en el trabajo $(p>0,05)$. Abrajan, Contreras y Montoya (2009) en México afirma que a mejor índice de favorabilidad, mayor nivel de satisfacción laboral. En Perú, Murrugarra (2017) observó que solo la dimensión aspectos psicosociales de la variable características ergonómicas laborales (Rho 0,278, p $0,001)$ se asoció con la satisfacción laboral. Del Campo (2014) que el derecho a la vida es la esencia del derecho a la salud y como tal inexigible a la persona-trabajador. Añanca (2014), en Lima investigo que la intervención ergonómica disminuyó el riesgo laboral. Farfán (2014) propone medidas de seguridad para mejorar las condiciones del trabajo como prevención frente a riesgos laborales. Tipán (2012) fianlemnet afirma que la gestión del trabajo es un 
factor de aparecimiento de riesgos ergonómicos en personal cuyo puesto de trabajo implica el uso continuo de PVD, posturas adoptadas incorrectas, trabajo continuo sin pausas.

\section{OBJETIVO}

Determinar si existe relación en la ergonomía de los puestos de trabajo y la satisfacción laboral extrínseca e intrínseca del personal administrativo de la sede Rectorado de la Universidad Privada de Tacna en el 2018.

\section{METODOLOGÍA}

Es un estudio con un enfoque cuantitativo, correlacional se utilizará la prueba de Chi cuadrado en la Sede del Rectorado de la Universidad Privada de Tacna ubicada en el distrito de Tacna del 2018. Muestra de tamaño 64. Para fines del presente estudio, se seleccionó la escala de satisfacción OverallJob Satisfaction (Warr, Cook y Wal, 1979) y el cuestionario de características ergonómicas del puesto de trabajo para el personal que trabaja con pantallas de visualización de la INSHT. Se realizó una prueba piloto con 20 unidades de análisis para estimar la confiabilidad de los instrumentos de medición. Asimismo, durante la fase de levantamiento de información en el ámbito de estudio, se cumplió con los procedimientos administrativos y éticos establecidos para la investigación. Para el análisis univariado se recurrió a la estadística descriptiva y para la contrastación de las hipótesis a la prueba estadística de Chi cuadrada.

\section{RESULTADOS}

Se evidencia que la edad que predomina es de 20 a 30 años seguido del grupo de personal que tiene entre 21 a 40 años, de 50 años a más (21,3\%) y el personal que tiene entre 40 a 50 años $(19,7 \%)$. En general el $78,8 \%$ tiene 50 años o menos. En lo que respecta al género son mujeres $(57,4 \%)$ y hombres $(42,6 \%)$. En lo que atañe al estado civil, predomina el grupo de solteros/as con un $55,7 \%$, seguido de los casados/as con un $37,7 \%$. Es contratado $(50,8 \%$ ), y nombrado (49,2\%). Según tiempo de servicios, de 0 a 5 años representó el 50,8\%, seguido del grupo que presenta de 15 a más años de servicios.

Según se aprecia que las características ergonómicas de la posición del teclado presentan en su mayoría, en todos los casos más del $50 \%$ de cumplimiento de las características de diseño de posición del teclado, especialmente la correcta disposición de las articulaciones del codo aproximadamente a $90^{\circ}(68,9 \%)$ y cuando se admite el antebrazo cuando se una un mouse u otro dispositivo portátil $(67,2 \%)$.

La evaluación de las características ergonómicas referida a si la parte superior de la pantalla del monitor queda ubicada a la altura de los ojos solo un $68,9 \%$ se cumple. La característica ergonómica de los asientos en el trabajo que se cumple en mayor medida es "los muslos son aproximadamente paralelos al suelo"(77\%), "el borde superior del respaldo está a 45-55 $\mathrm{cm}$ por encima del asiento o respaldo estándar" $(70,5 \%)$, igualmente la característica ergonómica " los pies están planos en el piso o se proporciona un reposapiés donde sea (70,5\%) y la altura del plato del asiento es ajustable de 42 a $51 \mathrm{~cm}$ (asiento estándar) $(68,9 \%)$, lo que resulta favorable y satisfactorio para la prevención de riesgos laborales o 
enfermedades ocupacionales. Sin embargo, la característica en cuanto a "la altura del soporte lumbar es ajustable de 15 a $25 \mathrm{~cm}$ por encima del asiento" en un 83,6\% es una condición que no se cumple , al igual que los asientos con un "ángulo del respaldo ajustable $93-113^{\circ}$ " en un 73,8\%. Lo mismo ocurre, con la "inclinación del asiento es ajustable de +3 a $-4{ }^{\circ \prime \prime}$ que en un $62,3 \%$ de los asientos tampoco se cumple. Los asientos presentan confort para la mayoría de los trabajadores, sin embargo, en muchos casos estos no cumplen con las condiciones ergonómicas estándar según si el trabajador es hombre o mujer, lo que determina condiciones y exigencias distintas dadas las diferencias anatómicas.

En cuanto a las características de los escritorios, es importante mencionar que en la mayoría de los casos la característica ergonómica deseable de un "espacio horizontal de la rodilla es mayor de 43 centímetros" se cumple en un $85,2 \%$ de los casos, y en menor medida "el espacio horizontal de la punta sea mayor de 60 centímetros" con un 65,6\%, lo que evidenciaría que prácticamente la tercera parte es una condición que no se cumple. Los diseños del escritorio generalmente responden a criterios tradicionales, además de que, en ciertos casos, están ubicados en espacios reducidos, situación que resulta de incomodidad sobre todo para los trabajadores de la rama de tecnologías de la información.

Dado que el uso de condiciones ergonómicas en el personal estudiado, comprende generalmente el uso de pantallas de visualización, se observó que en un 90,2\% no se cuenta con un soporte de documentos ajustable. Igualmente, en el caso que, si lo tiene, tres personas respondieron que es lo suficientemente grande para los documentos que utiliza $(4,9 \%)$ y la diferencia comprende a quienes consideran que no lo es y los que no poseen un soporte para los documentos $(95,1 \%)$. Cabe subrayar, que el personal en su mayoría no cuenta con un soporte de documentos ajustable, lo que dificulta la manipulación de documentos y en muchos casos obliga a adoptar posturas inadecuadas que podrían causar lesiones o algias dorsales o cervicales. (tabla 01).

Tabla 1

Ergonomía del puesto de trabajo

\begin{tabular}{|c|c|c|c|c|}
\hline & \multicolumn{2}{|c|}{$\mathrm{Si}$} & \multicolumn{2}{|c|}{ No } \\
\hline & $\mathrm{N}^{\circ}$ & $\%$ & $\mathrm{~N}$ & $\%$ \\
\hline 1.Los brazos superiores cuelgan relajados al costado durante el uso de la computadora. & 40 & 65,6 & 21 & 34,4 \\
\hline 2.Las articulaciones del codo están a aproximadamente $90^{\circ}$. & 42 & 68,9 & 19 & 31,1 \\
\hline 3.Las manos están en línea con los antebrazos cuando se usa el teclado y / o el mouse & 36 & 59,0 & 25 & 41,0 \\
\hline 4.Se admite el antebrazo cuando se usa un mouse u otro dispositivo portátil & 41 & 67,2 & 20 & 32,8 \\
\hline 5.Tanto la altura del teclado como del mouse permiten posturas de brazo apropiadas & 39 & 63,9 & 22 & 36,1 \\
\hline $\begin{array}{l}\text { 6. La parte superior de la pantalla está a la altura de los ojos (excepto los usuarios } \\
\text { bifocales y trifocales) }\end{array}$ & 51 & 83,6 & 10 & 16,4 \\
\hline 7. La distancia de visión (ojos a pantalla) es de 40 a $74 \mathrm{~cm}$. & 47 & 77,0 & 14 & 23,0 \\
\hline 8. El monitor está centrado frente al usuario durante el uso continuo de la computadora & 42 & 68,9 & 19 & 31,1 \\
\hline 9. La altura del plato del asiento es ajustable de 42 a $51 \mathrm{~cm}$ (asiento estándar) & 42 & 68,9 & 19 & 31,1 \\
\hline 10. La inclinación del asiento es ajustable de +3 a $-4^{\circ}$ & 23 & 37,7 & 38 & 62,3 \\
\hline 11. La altura del soporte lumbar es ajustable de 15 a $25 \mathrm{~cm}$ por encima del asiento & 10 & 16,4 & 51 & 83,6 \\
\hline 12. El ángulo del respaldo al asiento es ajustable $93-113^{\circ}$ & 16 & 26,2 & 45 & 73,8 \\
\hline $\begin{array}{l}\text { 13. El borde superior del respaldo está a } 45-55 \mathrm{~cm} \text { por encima del asiento (respaldo } \\
\text { estándar) }\end{array}$ & 43 & 70,5 & 18 & 29,5 \\
\hline 14. El trabajador sabe ajustar la silla para mayor comodidad. & 37 & 60,7 & 24 & 39,3 \\
\hline 15. Los muslos son aproximadamente paralelos al suelo. & 47 & 77,0 & 14 & 23,0 \\
\hline 16. Los pies están planos en el piso o se proporciona un reposapiés donde sea necesario & 43 & 70,5 & 18 & 29,5 \\
\hline 17. El espacio horizontal de la rodilla es mayor de $43 \mathrm{~cm}$. & 52 & 85,2 & 9 & 14,8 \\
\hline 18. El espacio horizontal de la punta es mayor de $60 \mathrm{~cm}$. & 40 & 65,6 & 21 & 34,4 \\
\hline 19. Un soporte de documentos ajustable está presente & 6 & 9,8 & 55 & 90,2 \\
\hline 20. El soporte es lo suficientemente grande para los documentos que se utilizan. & 3 & 4,9 & 58 & 95,1 \\
\hline
\end{tabular}


De las observaciones realizadas, las condiciones ergonómicas de iluminación que se cumplen mayormente, son los niveles de iluminación entre 300 y 500 lux (70,\%), el área de oficinas está iluminada con luminarias indirectas $(34,4 \%)$ y la línea de visión del personal es paralela al plano de las ventanas (39,3\%). No obstante, es relevante merituar que las condiciones ergonómicas de pantalla de ordenador libre de reflejos en su mayoría no lo están (77\%), las lámparas de techo cuentan con filtros parabólicos $(93,4 \%)$ y el trabajador cuenta con una luz de trabajo ajustable $(90,2 \%)$ si en caso correspondiese. (Fig. 1)

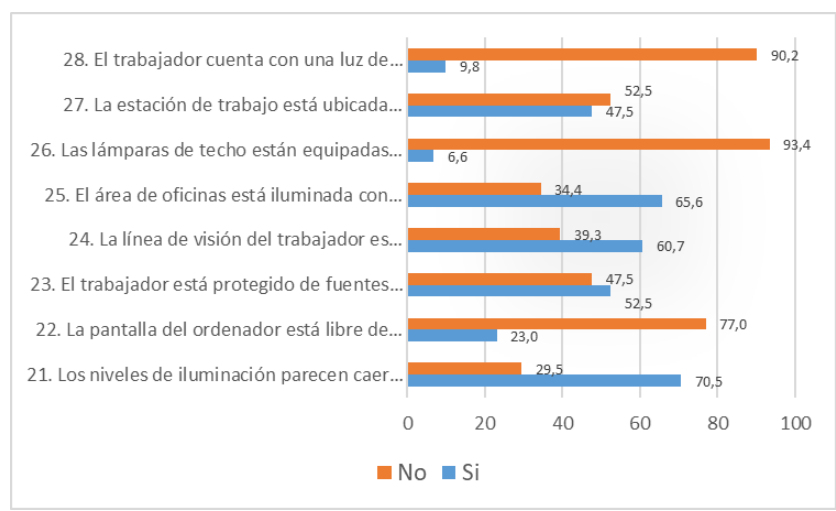

Figura 1. Características ergonómicas de iluminación

En lo que concierne a los descansos recomendados para quienes utilizan durante una larga jornada el teclado, por ejemplo, cinco minutos cada hora, la mayoría manifiesta que no lo hace $(83,6 \%)$. No se ha considerado disposición alguna en reglamentos, directivas u otros documentos normativos, sobre recomendaciones de descanso frecuente al trabajador que labora muchas horas en forma continuada frente a una pantalla de visualización de datos. (Figura 2)

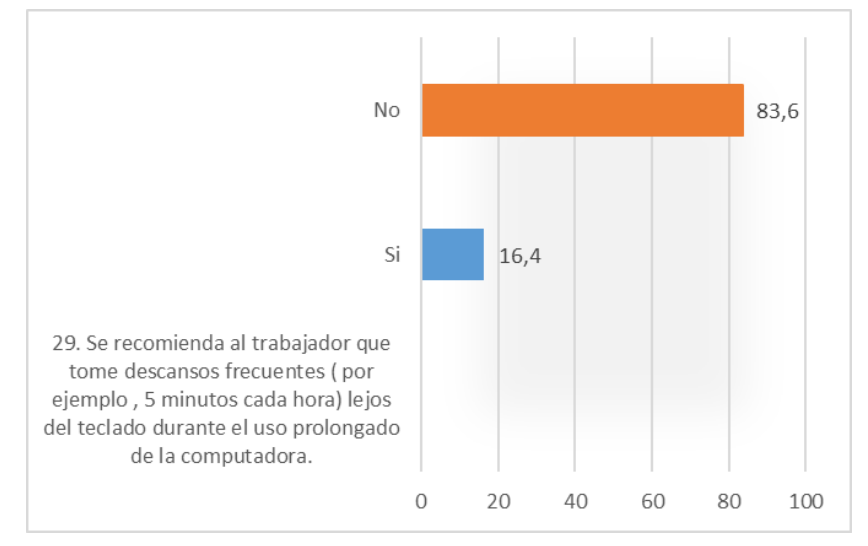

Figura 2: Características ergonómicas de diseño de tareas

\section{Satisfacción laboral}

De los ítems valorados para la satisfacción con los factores extrínsecos, el ítem mejor valorado es aquel que está referido a la dinámica laboral en relación con el "superior inmediato" (Promedio 3,84 en una escala de respuesta de 1 a 5), mientras que el ítem "salario" presenta la valoración más baja (Promedio de 2,85 en una escala de respuesta de 1 a 5). Asimismo, el ítem 1 referido a las condiciones de trabajo se ubica por encima del 
promedio $(3,34)$, con tendencia favorable hacia un estado de satisfacción; el ítem 7 que alude al horario de trabajo también se encuentra sobre el promedio $(3,59)$. (Figura 3 )

De los ítems valorados para la satisfacción con los factores intrínsecos, el ítem mejor valorado es aquel que está referido a "la responsabilidad que se le ha asignado al personal" (Promedio 3,43 en una escala de respuesta de 1 a 5), mientras que el ítem "posibilidades de promocionar" presenta la valoración más baja (Promedio de 2,98 en una escala de respuesta de 1 a 5). Es importante destacar el ítem 1 que refleja la satisfacción con la "libertad para elegir su propio método de trabajo" con un valor ligeramente por encima de la media de 3,20 , al igual que la posibilidad de utilizar sus capacidades en función de los recursos cognitivos sino operativos que presenta un valor también por encima de la media de 3,80, que también tiende a ser positivo o de satisfacción. (Figura 4)

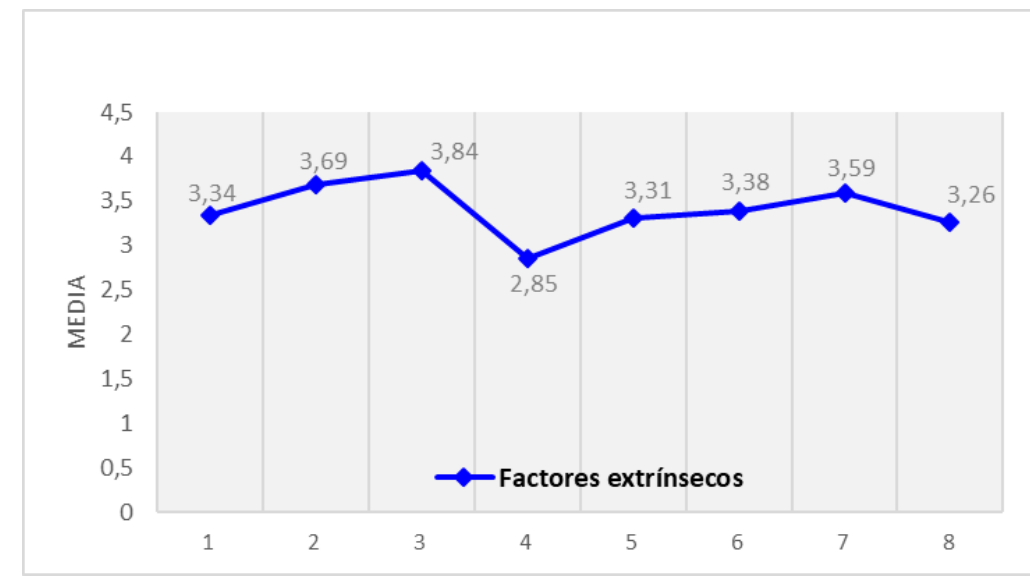

Figura 3. Factores extrínsecos

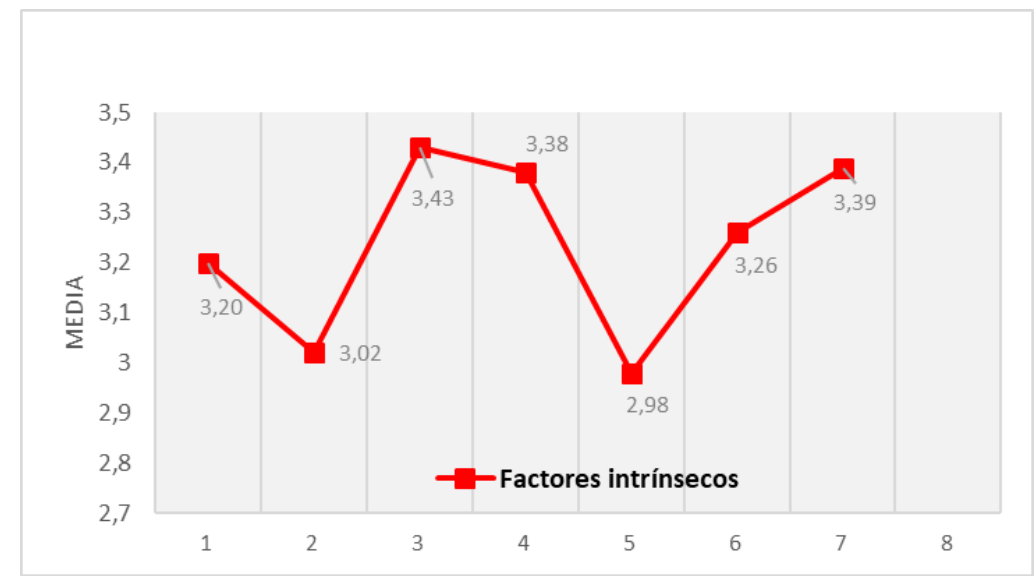

Figura 4. Factores intrínsecos

En lo que concierne a la relación entre la ergonomía del puesto del Principio de Prevención de la Ley $N^{\circ} 29783$ y la satisfacción laboral en general, se encontró que la asociación es significativa entre la característica ergonómica del puesto altura del plato del asiento ajustable de 42 a51 cm como medida estándar ( p 0,04; CCPawlik 0,255: débil asociación ), borde superior de respaldo entre 45 y $55 \mathrm{~cm}$ por encima del asiento ( $p$ 0,04;CCPawlik 0,255 : débil asociación ) y la recomendación de que el trabajador tome descansos frecuentes de 5 minutos entre horas, es decir, fuera del uso del teclado y de la computadora cuando la jornada de trabajo es larga ( 0 0,001;CCPawlik 0,386 : moderada asociación ). (Tabla 2) 


\section{Posición teclado}

1. Brazos superiores cuelgan relajados al costado al usar computadora.

2. Articulaciones del codo están a aproximadamente $90^{\circ}$.

3. Manos están en línea con antebrazos cuando se usa el teclado y/o mouse

4. Se admite el antebrazo cuando se usa un mouse u otro dispositivo portátil

5. Altura del teclado como del mouse permiten posturas de brazo apropiadas

$\begin{array}{rrr}0,098 & 0,754 & -- \\ 0,001 & 0,974 & -- \\ 0,713 & 0,399 & -- \\ 4,721 & 0,03 & -- \\ 1,209 & 0,272 & -- \\ 0,913 & 0,339 & -- \\ 0,0 & 0,990 & -- \\ 0,502 & 0,479 & --\end{array}$

\section{Posición monitor}

6. Parte superior de la pantalla está a la altura de los ojos

7. Distancia de visión (ojos a pantalla) es de 40 a $74 \mathrm{~cm}$.

8. Monitor centrado frente al usuario durante uso continuo de computadora

\section{Asientos}

9. Altura del plato del asiento es ajustable de 42 a $51 \mathrm{~cm}$ (asiento estándar)

10. Inclinación del asiento es ajustable de +3 a $-4{ }^{\circ}$

$4,238 \quad 0,040 \quad 0,255$

11. Altura soporte lumbar ajustable de 15 a $25 \mathrm{~cm}$ por encima del asiento

$0,338 \quad 0,561$

$0,538 \quad 0,463$

12. Ángulo del respaldo al asiento es ajustable $93-113^{\circ}$

$1,277 \quad 0,258$

13. Borde superior del respaldo está a $45-55 \mathrm{~cm}$ por encima de asiento

14. Trabajador sabe ajustar la silla

15.Muslos son aproximadamente paralelos al suelo.

16. Los pies están planos en el piso o con reposapiés donde sea necesario

\section{Escritorios}

17. Espacio horizontal de la rodilla es mayor de $43 \mathrm{~cm}$.

18. Espacio horizontal de la punta es mayor de $60 \mathrm{~cm}$.

$4,238 \quad 0,040 \quad 0,255$

$1,456 \quad 0,228$

$0,0 \quad 0,990$

$1,584 \quad 0,208$

Titulares de documentos

19. Soporte de documentos ajustable está presente

20. Soporte de documentos es lo suficientemente grande

\section{Iluminación}

21. Niveles de iluminación parecen caer entre 300 y 500 lux.

22. Pantalla del ordenador está libre de reflejos.

23. Trabajador e protegido de fuentes de deslumbramiento directo.

24. Línea de visión del trabajador paralela al plano de las ventanas.

25. Área de oficinas iluminada con luminarias indirectas.

26. Lámparas de techo e equipadas con filtros parabólicos

27. Estación de trabajo e ubicada entre filas de luces superiores.

28. Trabajador cuenta con una luz de trabajo ajustable (si es necesario)

$0,005 \quad 0,942$

$2,654 \quad 0,103$

$0,574 \quad 0,449$

$0,272 \quad 0,602$

$3,780 \quad 0,052$

$0,571 \quad 0,450$

$1,863 \quad 0,172$

$0,509 \quad 0,476$

$0,943 \quad 0,332$

$2,101 \quad 0,147$

$0,546 \quad 0,460$

$0,574 \quad 0,449$

\section{Diseño de tareas}

29. Trabajador toma descansos frecuentes (Ej. $5^{\prime}$ cada hora) lejos de teclado 


\section{DISCUSIÓN}

La presente investigación estudió la relación entre la ergonomía del puesto de trabajo del principio de prevención de la Ley $N^{\circ} 29783$ y su relación con la satisfacción laboral del personal administrativo de la sede rectorado de la Universidad Privada de Tacna.

Es necesario subrayar, que la Ley $N^{\circ} 29783$ Ley de Seguridad y Salud en el Trabajo publicada el 27 de octubre de 2016 en nuestro país, tiene como objetivo primordial promover una cultura de prevención de riesgos laborales. En este sentido, los hallazgos en el presente estudio permiten identificar condiciones adecuadas que cumplen las características ergonómicas, pero en algunos casos y en ciertas características del puesto de trabajo, son disergonómicas lo cual incrementa el riesgo de ocurrencia de enfermedades ocupacionales en el personal, aumento de ausentismo laboral y elevación de los costos sociales por parte del empleador. En relación a la variable ergonomía del puesto de trabajo, se encontró que las características del puesto de trabajo del personal que trabaja en las oficinas con pantallas de visualización de datos, en su mayoría responden a criterios ergonómicos que disminuyen la probabilidad de riesgos para la salud del personal, son la posición del teclado que en todos los indicadores (posición de los brazos para articular el uso de la computadora, la articulación del codo a $90^{\circ}$ aproximadamente, posición del antebrazo al utilizar el mouse u otro dispositivo y posición apropiada del brazo según la altura del teclado y mouse) presentan más del $58 \%$ de cumplimiento. No obstante, en varios casos, las condiciones no son adecuadas, por lo que siempre existe la posibilidad de riesgo de ocurrencia o exacerbación de alguna dolencia. Resulta también satisfactorio, los resultados encontrados en cuanto a las condiciones ergonómicas de la posición del monitor (parte superior de la pantalla a la altura de los ojos, distancia de visión ojos a pantalla de 40 a $74 \mathrm{~cm}$ y monitor centrado frente al usuario) en la gran mayoría de los casos cumple con las características ergonómicas en más del $68 \%$. Igualmente, se subraya, que aunque es una minoría de los usuarios, los que no presentan condiciones ergonómicas, la situación plantea la posibilidad de aparición de malestar o compromiso de su salud a largo plazo.

En lo que se refiere a las características ergonómicas de los asientos (altura del plato del asiento ajustable, altura del soporte lumbar, ángulo del respaldo, borde superior del respaldo, posición de los muslos paralelos al suelo, uso o disponibilidad de reposapiés) el cumplimiento es dispar, ya que en algunos casos como la altura del soporte lumbar ajustable de 15 a $25 \mathrm{~cm}$, las condiciones ergonómicas alcanzan un porcentaje de 16,4\% y asientos con borde superior del respaldo de $45-55 \mathrm{~cm}$ por encima del asiento en el 70,5\% se cumple en general, sin hacer distinción si el usuario es hombre o mujer, condición biológica que podría generar necesidades diferentes al estándar mencionado. Estos resultados, nos permiten presumir que las condiciones disergonómicas de los asientos respecto de algunas características podrían generar a futuro o exacerbar dolencias o algias. En lo referente a los escritorios, la condición ergonómica referida al espacio horizontal de la rodilla mayor a 43 $\mathrm{cm}$ se cumple en el 85,2\%, mientras que el espacio horizontal de la punta mayor de $60 \mathrm{~cm}$ solo se presenta en el $65,6 \%$, la diferencia correspondería a características disergonómicas que podrían generar dolencias o golpes durante el desplazamiento o movimientos necesarios durante el cumplimiento de la tarea o función. Otro aspecto, observado son las características de la manipulación y uso de los documentos de trabajo, para lo cual se recomienda como característica ergonómica saludable el uso de un soporte de documentos ajustable, condición reportada solo por el 9,8\% de los entrevistados. En tal sentido, es probable que la utilización para digitar en la pantalla los documentos pertinentes, la posición 
del usuario sea inadecuado, con una curvatura forzada o desplazamiento lateral del torso que puede generar dolor o en un largo plazo trastornos en la columna vertebral.

Es importante, también mencionar otro factor laboral indispensable como es la iluminación, encontrándose que solo una minoría con un $23 \%$ manifestó que la característica ergonómica de trabajar frente a una pantalla libre de reflejos se cumple. Igualmente, solo en el 6,6\% de los casos, los usuarios trabajan con lámparas de techo equipadas con filtros parabólicos y un 9,8\% manifiesta que cuenta con una luz de trabajo ajustable para realizar las tareas de oficina. En las demás características como nivel de iluminación (70,5\%), área de oficinas iluminadas con luminarias indirectas $(65,6 \%)$, el porcentaje de cumplimiento fue más alto. Es evidente, que, en la categoría de iluminación, las condiciones disergonómicas del puesto de trabajo, son más altas que las condiciones adecuadas en varias categorías, lo cual requiere mejoras para prevenir dolencias en los ojos, como cansancio visual, ojo seco u otra dolencia. También es necesario, mencionar que se recomienda que el personal que está muchas horas en un puesto de trabajo con tareas repetitivas o no, tome descansos frecuentes, lejos de las PVD. De los hallazgos, se advierte que los principales riesgos de salud que pueden determinar o acentuar problemas de salud en el personal estudiado que se desempeña en oficinas administrativas, están relacionados principalmente con el uso permanente de la pantalla de visualización de datos sin periodos de descanso, características disergonómicas del uso del teclado y otros dispositivos de entrada, la forma de manejo de los documentos, las características de la superficie o la mesa de trabajo, las características de los asientos, la iluminación, los momentos de descanso en el desarrollo del trabajo diario. Resultados similares reportan Andrango y Tituaña (2016) en Quito como resultado de la identificación y evaluación de riesgos ergonómicos en una empresa. En general, las características disergonómicas identificadas incrementan el riesgo de ocasionar alteraciones visuales como fatiga visual, fatiga física muscular (algias de cuello y nuca, dorsalgias, lumbalgias, contracturas, hormigueos, astenia, síndrome del codo, síndrome del túnel carpiano por inflamación del nervio mediano), en coincidencia Tipán (2012) también encuentra en su estudio condiciones desfavorables, e indica que a pesar de que el organismo humano en muchos casos es capaz de recuperarse por sí mismo, pero persisten las condiciones desfavorables de las condiciones del puesto de trabajo u organizacionales incorrectas, las consecuencias en la salud son inevitables.

Por otra parte, otro elemento que refleja las condiciones del entorno laboral es la satisfacción laboral, habiéndose encontrado que la media de la puntuación se ubica por encima del promedio de la escala, lo que evidencia una tendencia hacia la satisfacción, lo cual es favorable para el trabajador, ya que no solo promueve la productividad en la organización, sino que como elemento de salud mental, se considera un factor protector frente a condiciones de estrés laboral, que pueden también perjudicar la salud del personal en su entorno laboral. Cabe resaltar que la satisfacción laboral es un constructo opuesto y protector frente al síndrome de burnout o síndrome de quemarse en el trabajo y que es producido por el estrés que causa el trabajo generalmente en situaciones de insatisfacción laboral y otros elementos concurrentes.

En tal sentido, es importante también considerar la satisfacción en el trabajo, como un factor protector frente al estrés que puede causar la actividad laboral. Los hallazgos en el presente estudio revelan que en general la satisfacción laboral general presentó una media global de 49,9 en una escala de 15 a 75, tendiente a la satisfacción. Asimismo, los valores promedios de los factores extrínsecos son ligeramente más altos que los valores intrínsecos, lo que reflejaría que existe una mayor satisfacción extrínseca sobre aspectos vinculados con 
el puesto de trabajo como los horarios de permanencia, las condiciones físicas del trabajo, salario, dirección, etc. (diferencia escasa) respecto de los factores relacionados con el propio puesto de trabajo, a diferencia de la satisfacción intrínseca que alude al puesto en el que se desenvuelve. Por el contrario, Murrugarra (2017) encontró que la autopercepción de las condiciones ergonómicas en la dimensión aspectos psicosociales se asoció con la satisfacción laboral, más no con otras condiciones ergonómicas. Cabe precisar que, en el presente estudio, no se abordaron aspectos psicosociales.

A modo de colofón, a partir de la fundamentación jurídica es importante destacar, que la promoción de un ambiente de trabajo saludable y seguro, es un objetivo permanente de acción de la Organización Internacional del Trabajo (Ulloa citado por Sanguineti, 2009). Por ello, los resultados evidencian que existen características disergonómicas del puesto de trabajo en el ámbito de estudio, que deben ser mejoradas, a fin de garantizar condiciones que protejan la salud y el bienestar del personal. El fundamento jurídico se asienta en la Ley №29783, que establece principios rectores como el de Prevención, en el que taxativamente se indica que comprende los factores sociales, laborales - condiciones del puesto de trabajo - biológicos como enfermedades ocupacionales de diversa índole, como dolor de espalda, dolor lumbar, cansancio ocular, etcétera, diferenciados según el sexo del trabajador, e inclusive incorporando la dimensión de género en la evaluación y prevención de los riesgos en la salud derivado de las condiciones o características laborales.

En la misma Ley $\mathrm{N}^{\circ} 29783$, según el Principio de Protección, los trabajadores tienen derecho a que el Estado y los empleadores aseguren condiciones de trabajo dignas que a su vez protejan un estado de vida saludable en el ámbito físico, psíquico o mental y social, de modo continuo. El trabajo debe desarrollarse en un ambiente seguro y saludable y las condiciones de trabajo deben ser compatibles con el bienestar y la dignidad de los trabajadores y ofrecer posibilidades reales para alcanzar sus metas personales (Ley $\mathrm{N}^{\circ} 29783,2016$ ). Al respecto Del Campo (2014) como resultado del análisis de la citada norma, establece que la exigibilidad jurídica del Derecho a la Seguridad y Salud en el Trabajo, se fundamenta en la obligación de los Estados parte de la OIT de ejecutar en su territorio, normativas, políticas y recomendaciones que permitan la implementación de los sistemas de seguridad y salud laboral para garantizar la seguridad y salud en el trabajo, siendo el sustento la dignidad del ser humano.

A las autoridades y Jefatura del área de Gestión del potencial humano y en lo que atañe al Comité de Seguridad y Salud laboral, para fines de prevención de riesgos de salud ocupacional, en atención al Principio de Prevención de la Ley de Seguridad y Salud en el Trabajo Ley $N^{\circ} 29783$, rediseñar los puestos de trabajo, especialmente, para el personal que trabaja permanentemente con PVD, realizar la adecuación de la ubicación del monitor, del teclado, orientar sobre la postura de sentado, implementar la colocación de porta documentos, rediseñar las características de las superficies de trabajo, sillas de trabajo, reposapiés, regulación de las condiciones ambientales de iluminación, prevención de la fatiga mental y estrés, considerar la posibilidad de descansos alternos de 5 minutos aproximadamente cada dos horas para el personal que utiliza PVD, en orden a prevenir la aparición o exacerbar dolencias como consecuencia de la actividad laboral, dentro del Principio de Prevención, que establece la posición de garante del empleador de asegurar medios y condiciones que protejan la salud y bienestar de su personal. Se propone a la alta dirección y al área de Asesoría Jurídica, la adopción de un Compliance de seguridad y salud en el trabajo para el cumplimiento de las prescripciones establecidas en la Ley de Seguridad y Salud en el Trabajo Ley $\mathrm{N}^{\circ} 29783$, en materia de prevención de riesgos laborales. El 
propósito de este procedimiento, es asegurar que la institución no cometa infracciones o delitos por incumplimientos legales, es decir, la finalidad del Compliance en materia de seguridad y salud en el trabajo es prevenir, detectar y gestionar los riesgos relacionados con el incumplimiento de la normativa en la materia. Asimismo, la incorporación de riesgos legales laborales por incumplimientos de las normas de seguridad y salud laboral, dentro de las estrategias de programas del compliance penal es fundamental y estratégico, ya que la correcta determinación y tratamiento puede suponer la atenuación o exención de la responsabilidad legal de la institución y de sus autoridades ante los hipotéticos incumplimientos.

\section{REFERENCIAS BIBLIOGRÁFICAS}

Abrajan,M., Contreras,J. Montoya, S. (2009). Grado de satisfacción laboral y condiciones de trabajo: una exploración cualitativa. Enseñanza e Investigación en Psicología [en linea] 2009, 14 (enerojunio): [En línea] Recuperado de en:<http://www.redalyc.org/articulo.oa?id=29214108> ISSN 0185-1594

Andrango, D. y Tihuaña, J.(2016). Identificación y evaluación de riesgos ergonómicos y psicosociales aplicando el método Rula Office y f-psico 3.1 en la empresa ST Servicios y propuesta de medidas preventivas para los factores significativos. [En línea] Recuperado de http://www.dspace.uce.edu.ec/handle/25000/7882

Añanca (2014). Intervención ergonómica de bajo costo para disminuir el nivel de riesgo postural en los trabajadores administrativos de la Facultad de Medicina Humana y Ciencias de la Salud, Universidad Alas Peruanas Lima - 2013 ( Tesis) Universidad Alas Peruanas [En línea] Recuperado de http://repositorio.uap.edu.pe/handle/uap/2110

Asensio, S., Bastante, J. y Diego, J. (2012). Evaluación ergonómica de puestos de trabajo, Madrid: Ediciones Paraninfo.

Boluarte, A.(2014).Propiedades psicométrics de la Escala de satisfacción laboral de Warr, Cook y Wall, versión en español. RevMedHered vol.25 no.2 Lima abr. 2014 [En línea] Recuperado de http://www.scielo.org.pe/scielo.php?script=sci_arttext\&pid=\$1018-130X2014000200005

Brunette J. (2003). Satisfacción, salud y seguridad ocupacional en el Perú [En línea] Recuperado de http://www.upch.edu.pe/faspa/images/doc/brunette\%202003.pdf

Calderón, O.(2014). Evaluación ergonómica de los puestos de trabajo de usuarios de pantalla de visualización de datos (pvd) en las oficinas administrativas de Guayaquil de Pacificard S.A ( Tesis ) Universidad de Guayaquil [En línea] Recuperado de http://repositorio.ug.edu.ec/handle/redug/4509

Collado, S. (2008). Prevención de riesgos laborales: Principios y Marco normativo. En Revista de Dirección y Administración de Empresas. Número 15, diciembre 2008

Comunidad Andina (2018). Normativa andina [En línea] Recuperado de http://www.comunidadandina.org/Normativa.aspx?GruDoc=07

Del Campo, T. (2014). La configuración del Derecho a la Seguridad y Salud en el trabajo, a partir de los elementos que conforman la Seguridad y la Salud en el Trabajo, su regulación normativa y su análisis en la Ley N²9783 (Tesis de Maestría) Pontificia Universidad Católica del Perú, Lima.

Díaz, M. (2014). Ergonomía y satisfacción laboral [En línea] recuperado de https://www.cerem.pe/eventos-y-seminarios/ergonomia-y-satisfaccion-laboral

EsSalud (2016). Enfermedades ocupacionales e higiene ocupacional [En línea] Recuperado de http://www.essalud.gob.pe/downloads/ceprit/MAYO_2016__ Enfermedades _Ocupacionales_e_Higiene_Ocupacional.pdf

Farfán, J. (2014). Gestión de seguridad e higiene ocupacional y la satisfacción laboral en la industria textil (Tesis de titulación) Universidad Nacional del Callao, Lima. 
Instituto de Seguridad y Salud Laboral de la Región de Murcia (2008) I Encuesta Regional de Condiciones de Trabajo. Región Murcia [En línea] Recuperado de http://www.carm.es/web/pagina?IDCONTENIDO=11699\&IDTIPO=60\&RASTRO=c721\$m3637

Instituto Nacional de Seguridad e Higiene en el Trabajo - Madrid (2015). Iluminación en el puesto de trabajo. Criterios para la evaluación y acondicionamiento de los puestos [En línea] Recuperado de http://www.insht.es/InshtWeb/Contenidos/Documentacion/Iluminacion\%20en\%20el\%20 puesto\%20de\%20trabajo.pdf

León, J. (2013). La ergonomía y su relación con la satisfacción del personal de una distribuidora automotriz de la ciudad capital (Tesis titulación) Universidad Rafael Landívar [En línea] Recuperado de http://biblio3.url.edu.gt/Tesario/2013/05/57/De\%20Leon-Juan.pdf

L'Institut Sindical de Treball, Ambient i Salut (2011) Condiciones de trabajo y salud [En línea] Recuperado de http://www.istas.net/web/index.asp?idpagina=2142

Manosalvas, C. y Manosalvas, L. y Nieves, J. (2015). El clima organizacional y la satisfacción laboral: un análisis cuantitativo rigurosos de su relación. AD-minister, núm. 26, enero-junio, 2015, pp. 5-15 Universidad EAFIT, Medellín, Colombia.

Medina, C. (s.f.). Principios y conceptos en seguridad y salud en el trabajo [En línea] Recuperado de http://www.usmp.edu.pe/recursoshumanos/concurso2013/pdf/dif-fia10.pdf

Mesia, C. (2004). Derechos de la persona. Dogmática Constitucional, Fondo Editorial del Congreso de la República: Lima.

Ministerio de Trabajo y Promoción del Empleo del Perú (2009) Boletín de Economía Laboral: Las condiciones de trabajo y sus efectos en la satisfacción laboral de los asalariados privados de Lima Metropolitana. Dinámica de la pobreza y transiciones de la oferta laboral en el Perú [En línea] http://www.mintra.gob.pe/archivos/file/estadisticas/peel/bel/BEL_39.pdf

Murrugarra, J. (2017). La ergonomía y satisfacción laboral de los trabajadores de la municipalidad distrital de Pachacamac, periodo 2016 (Tesis de Maestría) Universidad Cesar Vallejo, Trujillo.

Nogareda S., Bestratén M. (2011). El descanso en el trabajo (I): pausas [ En línea] Recuperado de http://www.insht.es/InshtWeb/Contenidos/Documentacion

/Ficheros/891a925/916w.pdf

Organización Internacional del Trabajo (2004). Estrategia global en materia de seguridad y salud en el trabajo. Conclusiones adoptadas por la Conferencia Internacional del Trabajo en su 91.a reunión, 2003 [En línea] Recuperado de https://www.ilo.org/safework/info/policydocuments/WCMS_154865/.../index.htm

Organización Internacional del Trabajo (2018). Perú [En línea] Recuperado de https://www.ilo.org/global/lang--es/index.htm

Real Academia Española (2014) Diccionario de la lengua española [En línea] Recuperado de http://www.rae.es/recursos/diccionarios/drae

Resolución Ministerial № 375-2008-TR (2008) Norma Básica de Ergonomía y de Procedimiento de Evaluación de Riesgo Disergonómico, Diario Oficial el Peruano del Perú, 28 de noviembre de 2008.

Rodríguez, C. (2009). Los convenios de la OIT sobre seguridad y salud en el trabajo: una oportunidad para mejorar las condiciones y el medio ambiente de trabajo. Buenos Aires: Oficina OIT.

Rodríguez, E. (2014). Incidencia de los Estándares de Riesgos Ergonómicos en la Salud del Personal Administrativo que realiza labores de secretaria en la oficinas de la Universidad Estatal Península de Santa Elena:Diseño de un plan de Prevención ( Tesis de Maestría) Universidad de Guayaquil [En línea] Recuperado de http://repositorio.ug.edu.ec/handle/redug/3833

Sanguineti, W. (2009). Estudios de derecho del trabajo y de la seguridad social. Homenaje a Javier Neves Mujica, Grijley: Lima.

Tipán, I. (2012). Evaluación de riesgos ergonómicos en puestos de trabajo con pantalla de visualización de datos en la Gerencia de Seguridad, Salud y Ambiente -EP PETROECUADOR ( Tesis de Maestría) Universidad San Francisco de Quito [En línea] Recuperado de http://repositorio.usfq.edu.ec/bitstream/23000/ 1395/ 1/103 708.pdf 